\title{
Habitat loss and range shifts contribute to ecological generalisation amongst reef fishes
}

\author{
Authors: Rick D. Stuart-Smith*1, Camille Mellin ${ }^{1,2}$, Amanda E. Bates ${ }^{3}$, Graham J. Edgar ${ }^{1}$
}

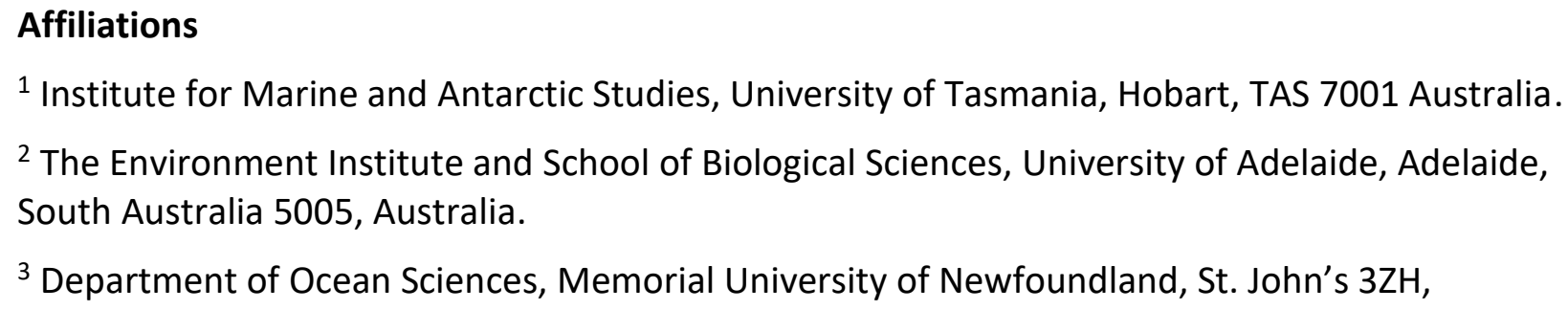

${ }^{3}$ Department of Ocean Sciences, Memorial University of Newfoundland, St. John's 3ZH, 10 Canada.

*Correspondence to: rstuarts@utas.edu.au.

\section{ABSTRACT}

Human activities are altering the structure of ecological communities, often favouring generalists over specialists. For reef fishes, increasingly degraded habitats and climate-driven range shifts may independently augment generalisation, particularly if fishes with least-specific habitat requirements are more likely to shift geographic ranges to track their thermal niche. Using a unique global dataset on temperate and tropical reef fishes and habitat composition, 20 we calculated a Species Generalisation Index (SGI) that empirically estimates the habitat niche breadth of each fish species. We then applied the SGI to evaluate potential impacts of habitat loss and range-shifts across large scales, on coral and rocky reefs. Our analyses revealed consistent habitat-induced shifts in community structure that favoured generalist fishes following regional coral mortality events and between adjacent sea urchin barrens and kelp habitats. Analysis of the distribution of tropical fishes also identified the SGI as the most important trait in predicting their poleward range extent, more so than body or range size. Generalist tropical reef fishes penetrate further into sub-tropical and temperate zones than specialists. Dynamic responses of reef fishes to habitat degradation imply loss of specialists at local scales, while generalists will be broadly favoured under intensifying anthropogenic pressures. An increased focus on individual requirements of specialists could provide useful guidance for species threat assessments and conservation actions, while ecosystem and multispecies fisheries models should recognise increasing prevalence of generalists. 


\section{MAIN TEXT}

Biotic homogenisation is considered a hallmark of human disturbance in natural ecosystems ${ }^{1}$, in part arising through replacement of ecological specialist species with generalists ${ }^{2}$. Compositional shifts towards communities dominated by generalists have been observed in birds and butterflies ${ }^{3,4}$, and are increasingly reported in marine systems ${ }^{5,6}$. Such shifts may already be a dominant form of ecological change on coral and rocky reefs around the world, particularly amongst the fishes, which represent an important link between reef habitats and human society ${ }^{7-9}$. Reef habitat degradation has become widespread through heatwaves and mass coral bleaching events ${ }^{10,11}$, devastating storms ${ }^{12}$, changing ocean climates ${ }^{13}$ and ecological impacts of fisheries ${ }^{14}$, each potentially intensifying habitat-related selective pressures on fishes.

Reshuffling of community structure is also occurring through species' range shifts in response to warming seas ${ }^{15}$, another process that may be influenced by species' ecological requirements, including habitat and diet ${ }^{16,17}$. For example, coral reefs are limited in their capacity to accrete rapidly enough to track warming seas ${ }^{18}$, raising concern about future mismatches between the potential distribution of coral reef fishes (with high range-shifting capability) and the distribution of coral reefs ${ }^{19}$. Habitat specialist fishes may be limited in their capacity to shift in distribution, while habitat generalists may be better able to colonise alternative habitats in sub-tropical and temperate zones.

Here we investigate the extent to which a loss of habitat-forming corals and kelps, along with species range-shifts, may together contribute to broad-scale generalisation of reef fish communities. We use an extensive global-scale dataset on co-located habitat and reef fish abundance data collected by the Reef Life Survey program ${ }^{20}$ to: (a) quantify global variation in reef habitat structure at local scales and identify reef fishes that occupy a large range of available habitats (generalists); (b) evaluate responses of reef fish communities to habitat loss for associated signals of generalisation, and; (c) test whether generalists are most likely to shift distributions as a result of their ability to occupy a broad range of habitats.

Rather than allocating coral reef fishes to pre-defined categories of habitat use (e.g. ${ }^{8,21}$ ), we applied a new approach to empirically estimate the extent that each species fills the range of reef habitats available across its entire geographic distribution. This method places species along a gradient from specialists to generalists, improving comparability, predictive capacity and allowing more nuanced community level analyses. First, habitat data from underwater photoquadrats were summarised into 16 broad benthic habitat categories (e.g. branching corals, algal turf, calcified algae, kelps; Extended Data 1) and all surveys globally were represented in 3-dimensional space using Principal Coordinates Analysis (PCO). The resulting 'habitat volume' captured $58 \%$ of the variation in local benthic habitat configurations observed on reefs surveyed around the world, from equatorial coral reefs to polar rocky reefs (Extended Data 2, 3).

We then calculated equivalent habitat volumes individually for 1,342 fish species, using data from the same transects along which habitat structure was quantified. Each species' habitat 
volume was expressed as the percentage of the habitat volume available to it (based on the total habitat volume from surveys across its geographic distribution). The resulting value is effectively an estimate of its habitat niche breadth, and is hereafter referred to as the Species Generalisation Index (SGI). Sites with no record of a species in the standardised surveys (i.e. absences) provided information about habitats apparently less suitable for that species. We applied a kernel probability density function rather than a convex hull to reduce the sensitivity of volume estimation to the number of records available, and also applied a lower cut-off of 70 records for species to be included in the analyses presented here. This choice did not alter conclusions from broad-scale and community level patterns but minimised the influence of species that may appear as specialists due to under-sampling. More information on volume estimation and tests of sensitivity to analytical decisions is provided in the Methods.

\section{Results and discussion}

Values of SGIs for reef fishes surveyed worldwide varied with species' body size, family and with geographic range size (Fig. 1), and were most closely related to their frequency of occurrence across surveys. Among reef fish families, the gobies (Gobiidae) were most dominated by specialists, while the larger families with many known specialists (e.g. Pomacentridae, Labridae and Serranidae) also included many generalists (Fig. 1a). Taxonomic family was therefore not an important predictor of SGI values. The significant relationship between SGI and frequency of occurrence ( $r=0.53, n=565, P<0.001$, two-tailed test) is to be expected if species with high SGI values are more often encountered across reefs as a result of low dependence on particular habitat characteristics. However, many other factors also contribute to how frequently species are encountered (e.g. competitive dominance, position in geographic range, fecundity, dispersal ability) and the extent to which these other drivers of frequency of occurrence contribute to SGI values likely varies considerably among species. While SGI values are calculated using habitat data, they should be interpreted with consideration that they may also reflect other elements of ecological generalisation (see Methods).

To assess the impacts of coral loss on coral reef fish communities, we calculated the Community Generalisation Index (CGI) as the mean of SGIs among species recorded on standardised quantitative fish surveys, weighted by the log of their abundance. We evaluated change in the CGI at 67 reef sites surveyed along the entire length of Australia's Great Barrier Reef before and after the mass coral bleaching event in 2016 (see ${ }^{22}$ ) and at 12 sites surveyed before and after a devastating cyclone on Ningaloo Reef in 2015. Coral loss was quantified on the same transects as the fish surveys, providing an explicit quantitative test for the effect of habitat change on CGI, unaffected by change in spatially variable factors such as wave exposure and depth.

The CGI generally increased at sites with greatest losses of live coral cover in both regions (Fig. 2a), although variation between sites in this response was high. CGI changes primarily related to species turnover, through both gains in generalist species and losses of specialists (Extended Data 4), rather than any coherent shifts in abundance of species relating to their SGIs. Gains in generalists may be assisted by greater frequency of occurrence, with more rapid postdisturbance colonisation possible if a species is more likely to live on adjacent reefs ${ }^{23,24}$. Yet, no 
significant trend was detected when frequency of occurrence was used instead of SGIs in an equivalent analysis (i.e. using a community-weighted mean of frequency values). Further testing of null models confirmed that the observed response was not likely to be simply related to how common species are, or any purely statistical properties of the SGI (see Methods). Decreases in CGI were also apparent at those sites where live coral cover increased between surveys (despite the bleaching or cyclone disturbance), indicating that rapid responses of specialists in the fish community are also possible during periods of coral recovery.

In temperate regions, habitats dominated by kelps and other canopy-forming macroalgae are threatened through human-mediated ecological processes (e.g. sea urchins and fishing interactions ${ }^{14}$ ) and changing ocean climate ${ }^{25}$. We assessed differences in the CGI of fish communities between macroalgal dominated habitats and their alternative, less complex 'barrens' habitats, using data from south-eastern Australia where sites spanning the full spectrum of these habitats are spatially interspersed in close proximity (see Methods). Fish communities at sites dominated by sea urchin barrens habitat possessed higher CGI values than at sites dominated by kelp, and the CGI increased significantly with the percentage of barren substrate across all sites (Fig. 2b, c). Thus, a transition to generalist species with increasing loss of kelps may be expected, although more rigorous testing with time-series data spanning kelp loss from multiple locations is needed to confirm the generality of this result.

Temperature appears to play a critical overarching role in shaping the latitudinal distribution limits of reef fishes ${ }^{26,27}$. Many tropical species have expanded poleward range margins with recent ocean warming, and temperate fish communities are becoming increasingly 'tropicalised'28. We evaluated the extent to which habitat requirements of tropical coral reef fishes may also influence their poleward range limits. We used the SGIs of 367 common tropical reef fishes recorded in Australia to test whether those found at higher latitudes along the north-south coastlines were more likely to be generalist species. We first recalculated SGIs after excluding records from temperate habitats to specifically test whether those that occupy a greater range of available habitat space in the tropics are also better able to colonise temperate habitats and extend further polewards (see Methods). After also removing juvenile vagrants that are sometimes common at higher latitudes ${ }^{29}, 71 \%$ of tropical reef fishes examined were recorded further south than the major areas of 'true' coral reef (i.e. accreted by reef-building corals; $\sim 24^{\circ} \mathrm{S}$. Fig. 3 ), often in rocky reef habitats with little or no coral cover. The tropical habitat SGI was clearly the most important predictor of observed species' southern latitudinal extent out of all the traits examined (Fig. 3b, Methods), which included maximum body size and geographic range size, both previously identified as key traits in distinguishing range shifting species ${ }^{15,30}$.

The SGI therefore appears to describe responses of reef fishes to abrupt habitat change and their ability to colonise new habitats as temperature and dispersal permit. Scaling species' responses up to the community-level with the CGI may provide a broadly applicable indicator of ecological change relating to habitat degradation, but further research is needed to investigate the processes that drive the non-negligible variation between sites, and to better understand the influence of spatial scale ${ }^{31}$. Our approach considers fish-habitat associations at the scale of 
$\sim 500 \mathrm{~m}^{2}$, but species interact with habitat features at different scales. Expert categorisations of coral dependence in reef fishes $8,21,32$ highlight finer-scale requirements for particular coral species or morphologies for larval settlement or food ${ }^{6}$, for example. The loss of such fishes at the patch scale ( 1-10 $\mathrm{m}$ ) may not become evident when using our SGI approach until enough patches are lost. Conversely, species' associations with giant kelp forests (Macrocystis pyrifera; which show catastrophic declines with warming ${ }^{33}$ ) likely require a slightly larger scale of investigation.

Our study raises questions relating to how specialists have responded to historical disturbances, and whether and how they will persist in future reef communities. Will specialists bounce back following acute disturbance events or will new specialist roles evolve? Or have historical rates and magnitudes of natural habitat and climate disturbances been exceeded, and are we now observing a process of extinction of specialists? Analysing trends in longer community timeseries (e.g. decades) spanning major disturbance events would provide important information on the longevity of community level responses such as those we observed for coral and kelp loss. Indeed, longer-time series from other ecosystems and taxa suggest replacement of specialists with generalists has been an important contributor to temporal beta-diversity patterns ${ }^{34,35}$. Such studies indicate that habitat specialism is probably not highly labile within species or readily developed over short periods ${ }^{36}$, and instead evolves over longer timescales. Thus, answering these questions likely requires understanding change over evolutionary, rather than ecological timescales.

Considering the two mechanisms addressed here for reef fishes, spatial heterogeneity in habitat loss and the rate of change in habitats through time are likely keys to the persistence of habitat specialists through geological timescales. Specialist persistence would be possible when habitat disturbances are patchy at scales small enough such that specialists could recolonise from patches of remaining suitable habitat during times of habitat recovery ${ }^{24}$. This was evident on an inter-annual timescale on the coral reefs we examined, where the CGI decreased (i.e. relative specialists became more prevalent), on average, at sites at which live coral cover increased between survey periods. Although one of the disturbance events in our analysis was a mass coral bleaching event that effected reefs globally, the impacts on reefs in the Great Barrier Reef study area were still highly patchy at local scales ${ }^{22}$. Likewise, damage from Cyclone Olwyn at Ningaloo in 2015 was extreme at some sites, but most of the surrounding areas of reef remained unaffected.

Based on the second mechanism, in which generalists better track their thermal envelope, persistence of specialists would only be supported when larger-scale changes in the distribution of habitats occurs concurrently with environmental change (i.e. when specialists can simultaneously track thermal and habitat niches through time and space). Coral reef and temperate kelp forest habitats have experienced dramatic reductions in global extent over millennia during periods of unsuitable environmental conditions and sea levels, and have been hypothesised to provide refugia for associated species during these periods ${ }^{37,38}$. Thus, specialists in contemporary communities have probably been able to effectively track habitats through recent geological history. 
The implications of our results for specialist reef fishes in the Anthropocene will therefore likely depend on whether (1) the spatial footprint of contemporary habitat loss exceeds that of historical habitat disturbances, and (2) the rate of environmental change exceeds the capacity of habitats to respond. The former appears to be occurring through an increasing frequency of disturbance events, as observed for mass bleaching events ${ }^{39}$ and storms ${ }^{12}$, and should be considered a likely mechanism to erode specialisation in reef fish communities at regional scales. The latter is suggested for coral reefs at least ${ }^{18}$, and it is thus possible that some specialist reef fishes may be limited by habitat needs in their ability to track their thermal niches with warming. In our analyses, this could be the case up to one third of the tropical fishes examined, and may extend to many more of the rarer species for which we did not have enough data to undertake this particular analysis (but also noting that a proportion of these nominal specialists may presently be restricted to tropical reefs for reasons other than habitat specialisation).

The remaining two-thirds of the tropical fishes we analysed appear well-equipped to track their thermal niche and persist in a diverse range of habitats. This includes some species considered highly dependent on live corals, for which corals growing on rocky reefs appear to provide the necessary food or settlement cues. Our results suggest that the distribution of these generalists is unlikely to be restricted by habitat, but their ecological success within their new distributions could be strongly shaped by habitat characteristics, presenting an important avenue for further study. Changes in local abundance were not a major contributor to the CGI change shown in Fig. 2, and other recent studies have reported unexpectedly high resilience of coral-dependent fishes to coral disturbance ${ }^{40}$. Yet in contrast to local temporal changes in habitat, the greater magnitude of habitat change possible over the large spatial scales through range shifts may be more likely to influence abundance. This is currently difficult to assess, complicated by thermal gradients ${ }^{41}$ and interactions with new species.

While our findings do not conclusively point to a process of long-term generalisation on the world's reefs, we provide substantial evidence for two mechanisms that independently appear to lead towards this outcome - and which are both increasingly observed on reefs globally. Future reefs dominated by generalists may provide some benefits and opportunities, including greater resilience to further disturbances. But the implications for ecosystem functioning are largely unknown. Many rare species are likely to be specialists ${ }^{7}$ and possibly also perform rare ecosystem functions ${ }^{42}$. Evaluating the likely outcomes of generalisation on reef fisheries production and the many social values derived from reefs is also an important goal 21,43 , which should be assisted with measures such as the SGI and CGI. When combined with constantly improving knowledge of the drivers of reef fish distribution and abundance, these indices offer enhanced predictive capacity for ecosystem and fisheries models, a better understanding of threat status to multiple pressures, and can thus contribute to improved management outcomes. 


\section{METHODS}

\section{Fish and habitat data}

Standardised data on fish community structure were collected from 12,956 50-m underwater transects by trained scientific and recreational divers participating in the citizen science Reef Life Survey (RLS) program, while benthic habitat data were from photoquadrats of the substrate scored for a subset of 4,070 of these transects (Extended Data 2, 3). Full details of census methods are provided elsewhere ${ }^{20,44}$, and an online methods manual (www.reeflifesurvey.com) describes all data collection methods. Data quality and training of

10 divers are detailed in ${ }^{20,45}$. All observed fish species were counted in $5 \mathrm{~m}$-wide transect blocks, separated into $2.5 \mathrm{~cm}$ size classes to $15 \mathrm{~cm}, 5 \mathrm{~cm}$ classes to $30 \mathrm{~cm}, 10 \mathrm{~cm}$ classes to $50 \mathrm{~cm}$ and $12.5 \mathrm{~cm}$ classes above. For these analyses, we excluded small fishes likely to be recently settled juveniles by removing all individuals in the $2.5 \mathrm{~cm}$ size class for species with a maximum body size $<25 \mathrm{~cm}$, and all individuals in the 2.5 and $5 \mathrm{~cm}$ size classes for all species with a maximum body size $\geq 25 \mathrm{~cm}$.

Habitat photoquadrat images were taken by divers every $2.5 \mathrm{~m}$ along each of the same transect lines, and later scored using a grid overlay of 5 points per image, 100 points per transect. Scores were initially done using a set of 50 morphological and functional groups aligned with the standard Australian hierarchical benthic classification scheme ${ }^{46}$, but were aggregated into 16 broader groups designed to be more comparable over larger scales for this study (Extended Data 1).

\section{Exploring the global reef habitat space}

We performed a Principal Coordinate Analysis (PCO) of the habitat data scored from the photoquadrats, with individual surveys as the statistical units and the mean \% cover of benthic categories as the response variables - a similar approach to that suggested by Guisan et al. ${ }^{31}$. The first 3 axes collectively explained 58\% of variation in the habitat data (PCO1 21.7\%; PCO2 $18.9 \%$; PCO3 $16.9 \%$ ), with $<10 \%$ explained by any subsequent axis. Using $10 \%$ variation as the cut off, we included the first three dimensions to represent the habitat space. We used the 'pco' function in the R package 'labdsv' to perform the PCO, using Bray-Curtis similarity.

\section{Calculating the Species Generalisation Index values (SGIs) and Community Generalisation Index (CGI)}

We calculated SGIs using a slightly modified version of the 'Trait Probability Density' (TPD) approach by Carmona et al. ${ }^{47}$, which uses a 3D kernel density estimation with the 'kde' function in the ' $k s^{\prime} \mathrm{R}$ package. Like the TPD approach ${ }^{47}$, we calculated the probabilistic 'habitat volume' occupied by a species, but we further expressed this as a proportion of the total volume of surveys across all ecoregions it was recorded in. We did this to better account for spatial differences in sampling intensity and potential bias in the habitat space available to each species as a result. Unlike other approaches (e.g. convex hulls) by which volume cannot decrease when additional samples are added, volumes calculated using TPD depend on the distribution of samples within the volume and can thus decrease if additional samples are highly clustered. We further applied a $90^{\text {th }}$ percentile cut-off, excluding the most extreme $10 \%$ of data points in the PCO cloud before calculating volumes so that vagrants in unusual habitats 
(outliers) did not contribute to the 'core' volume occupied by the species. We tested the sensitivity of SGI to this cut-off by recalculating SGI with cut-offs at the $85^{\text {th }}$ and $95^{\text {th }}$ percentiles and found these to be highly correlated with those based on $90^{\text {th }}$ percentiles $(r>0.99)$. Thus, our results are unlikely to be affected by the exact cut-off chosen.

The SGI is also, in theory, similar to the species specialisation index used for birds by Devictor et al. ${ }^{3}$, albeit with continuous description of habitat variation rather than categorical classification. We recognise the conceptual work of these authors, but deliberately chose not to use the same terminology here due to both the methodological differences and the uncertainty over whether low values represent true specialists or species that are rare for other reasons.

Our SGI is more appropriate for identifying generalists. We had a total of 1,342 fish species with enough data to calculate SGIs (using a minimum of 30 matching occurrence and habitat records each), but after preliminary data exploration, found the number of specialists to be disproportionately higher amongst species with fewer records. We therefore undertook a sensitivity analysis to identify the point at which adding more records for species did not appear to change the proportion of specialists identified. We did this by family, given some fish families naturally contain more specialists than others. We recalculated the family-level mean SGI values when including only species with more than 50, 70, 80, 90, and 100 records and plotted the relationships. Most families plateaued at 70-80 records, as also reflected in the overall mean trend among families, suggesting that 'sampling specialists' (i.e. those with low SGI as a result of inadequate sampling) were reduced in the dataset when including only species with more than $\sim 70$ records. We thus decided to restrict analyses for our major conclusions in Figures 1-3 to the 565 species with $>70$ records, for which we could be most confident that the proportion of 'false' specialists was minimal. Yet, as above, our major conclusions remain based on relative 'generalists', which should be much less sensitive to sample sizes.

For Figure $1 \mathrm{~b}$, a random forest analysis was run with $\mathrm{SGI}$ as the response variable and six species-level traits plus taxonomic family as predictors, using the packages 'randomforest' and 'party' in R. Traits used were geographic range size, frequency of occurrence, mean abundance, maximum length, water column position and trophic group, using values of the latter three traits from Stuart-Smith et al. ${ }^{48}$. Frequency of occurrence $(F)$ was calculated as the proportion of surveys a species has been recorded on out of all surveys within all ecoregions where it has been recorded. Mean abundance was calculated as the average density (per $500 \mathrm{~m}^{2}$ survey area) of the species whenever it was recorded. Geographic range size was calculated as in Stuart-Smith et al. ${ }^{26}$, as the combination of latitudinal and longitudinal extent, weighted by the density of presence records and corrected for global variation in the linear distances represented by one degree of latitude:

$$
\sqrt{\left(d_{v} \cdot \operatorname{stdev}(\text { Lat })\right)^{2}+\left(d_{h} \cdot \operatorname{stdev}(\text { Long })\right)^{2}}
$$

$40 \quad$ Where $d_{v}$ and $d_{h}$ are the distances (in $\mathrm{km}$ ) of one degree of latitude and longitude for the midpoint of a species' range, respectively, and stdev(Lat) and stdev(Long) the standard deviations of latitudes and longitudes of all occurrence locations for that species. Maximum 
correlation among the numerical traits use was -0.34 (between range size and $F$ ), and the variance in the SGI explained by all seven traits was $42 \%$.

The Community Generalisation Index (CGI) was calculated for each standardised RLS fish survey, using data on all fish species recorded, after removing mobile pelagic species not closely associated with the reef and species with SGIs based on fewer than 70 records (as above). We used the 'weighted.mean' function in R, weighting the mean SGIs of species recorded on the transect by $\log ($ density +1$)$.

\section{Temporal change and distribution limits analyses}

The response of reef fish communities to coral loss was evaluated at the Great Barrier Reef (GBR) and Ningaloo Reef using data collected by RLS divers before and after the 2016 mass coral bleaching event and Cyclone Olwyn in 2015, respectively. The GBR sampling design is described in Stuart-Smith et al. ${ }^{22}$, with surveys at each site divided in three depth bins $(<4 \mathrm{~m}, 4$ $10 \mathrm{~m},>10 \mathrm{~m}$ ) and unique site-by-depth bin combinations used as replicates. For Ningaloo, data came from the 12 sites surveyed in both 2012 and 2016 with complete coral cover data available in photoquadrat scores at the time of the study. In both cases, coral cover change is expressed per site as the absolute \% change in total live hard corals. A linear mixed effects (LME) model was run with CGI change as the response variable, \% coral cover change, location (Ningaloo or Great Barrier Reef) and the interaction of coral cover change and location as fixed effects, and reef as a random effect to account for variation along the length of the GBR. Figure 2 trends show linear fits for CGI change as a function of coral cover change (model summary outputs are reported in Extended Data 5).

Data for Figure 2(b, c) were all RLS surveys with scored photoquadrat scores available from Jervis Bay and Batemans Bay regions (New South Wales, latitude $35^{\circ} \mathrm{S}$ to $36.3^{\circ} \mathrm{S} ; n=123$ surveys), where the habitat varies enormously from site to site as a result of destructive grazing of the sea urchin Centrostephanus rodgersii. A PCO using Bray-Curtis dissimilarity of the habitat category cover data was used to distinguish sites as either sea urchin barrens or kelp habitats (representing opposite ends of PCO1, which explained $45 \%$ of total variation in benthic cover). Many sites in close proximity could safely be assumed to experience the same environmental conditions, only differing in the relative cover of kelp vs. barrens, providing an ideal opportunity to evaluate the effects of these alternative habitats on the fish communities. The distribution of CGI values for fishes recorded on surveys classified as having either 'kelp' or 'barrens' habitat are shown in boxplots (Figure 2 b). Further to this, the CGI of the fishes at all sites in the region was analysed in relation to the percentage of bare habitat categories (the sum of 'non-living', 'calcified algae' and 'encrusting' cover) using a Generalised Least Squares model in R. Figure 2c shows the significant linear fit for CGI as a function of barrens cover within sites (model summary outputs are reported in Extended Data 5).

The poleward latitudinal limit of tropical species used in Figure 3 was calculated as the southern-most record for each tropical species recorded in Australian waters with more than 70 records available to calculate SGIs (as above). Tropical species were defined on the basis of their distributions, with thermal midpoint $>23^{\circ} \mathrm{C}$, as in $(24,41)$. Juveniles were excluded using 
the criteria above and SGI was recalculated for each species based on tropical surveys only (i.e. only including sites in tropical realms ${ }^{49}$ ). We used only tropical sites for this test to remove the contributions of the temperate habitats to their SGI. This effectively de-couples their SGI values from the occupation of temperate zones and allows a more conservative and robust test of the prediction that their occupation of temperate zones is assisted by their capacity to occupy a greater range of habitats. A random forest analysis was first used to evaluate the importance of species traits as predictors of species' poleward range limits and narrow down the number of predictors for subsequent analysis. This was run on all traits listed above, plus the tropical SGI, and found the tropical SGI to be clearly the most important predictor, with a relative importance score of more than twice that of any other trait. A linear mixed effects model (LME) was then used to account for taxonomic family as a random effect (to control for the nonindependence of fish response due to phylogenetically conserved life history parameters such as fecundity and dispersal ability) with tropical SGI, frequency of occurrence, abundance and maximum length as fixed effects. The final model thus evaluated the southern range limit of 367 tropical reef fishes as a function of the continuous traits (pseudo $R^{2}=0.22$ ). Standardised estimates and the $95 \%$ confidence intervals from the LME are provided in Fig. 3. Basemaps for Figure 3 and Extended Data 2 were obtained using the R 'mapdata' package ${ }^{50}$, under the GNU General Public Licence 2.

\section{Interpretation of SGIs and CGI}

As described in the main text, SGIs were correlated with the frequency of occurrence $(F)$ of each species and should therefore be interpreted with consideration of other forms of ecological generalisation in addition to that associated with habitats. However, SGIs clearly contain more information than $F$ with respect to changing habitats and poleward latitudinal limits as explored in this study. We compared the trends in CGI values associated with coral loss in Figure $2 a$ with two levels of null models. The first considered only the statistical effect of $F$ and involved calculating the equivalent community weighted mean for each survey using $F$ instead of SGI (i.e. a community weighted frequency of occurrence index). This was not significantly related to coral change when substituted into the same LME (coefficient $=-0.005$, $p=0.94)$. The second null model was more complicated, involving resampling the original survey data to calculate null SGIs for each species in a way that reflected their $F$, but randomised with respect to the habitat cover. In other words, each species was randomly allocated to surveys (and therefore habitat data) within its geographic range, according to the proportion of sites it occurs on. This was repeated 100 times to generate a null distribution of SGI values for each species, with the mode of these used as a null SGI value in recalculation of the CGI and LME, as done for $F$ above. The effect of coral cover change on the null CGI was significant, but the effect size was two orders of magnitude smaller than when using our original CGI values (based on SGIs that linked species to observed habitats; coefficient $<-0.001 p=<0.01$ ). Thus, our observations using CGI reflect important links between species' responses to coral loss and their occupation of habitats by species across their entire ranges that $F$ does not capture. In terms of describing southern latitudinal limits of tropical species, the importance and unique contribution of SGIs was very clear. The relative importance (random forest analysis) and standardised effect size (LME) of tropical SGI were considerably larger than those for any other 
trait assessed, and the LME model found the effect of $F$ to be not only weaker, but also in the opposite direction (Fig. 3).

There are important sources of error in SGIs and CGIs which are presently unaccounted for. Specialists in our data tend to also be rare, and therefore a disproportionate number of specialists are likely excluded due to inadequate data. Likewise, endemic species restricted to areas with limited habitat variability may appear more specialised (e.g. those found only in the middle of the Coral Triangle, where survey sites largely consisted of relatively healthy coral habitat). Our approach also misses non-reef habitats occupied by species. To some extent species that occupy seagrass, soft sediments and mangroves, for example, may also occupy a greater range of reef habitat types, and will consequently have a larger SGI. Yet this assumption has not been tested and will be an interesting avenue for further research, when such data are available. Some species have also been shown to alter habitat use with changing ambient temperatures ${ }^{51}$, and our approach does not account for intra-specific variation that may influence habitat associations at different parts of the geographic range or in different seasons. Finally, we have not accounted for the disturbance history of survey sites used for the calculation of SGIs. The assumption is that a species no longer occurs in the degraded habitat following disturbance, so does not show up in our data if the site is no longer suitable (i.e. we assume no lag effects where a species may remain for some time after the disturbance). Given the rapid reshuffling of communities shown in Fig. 2a, we consider this a reasonable assumption. But such lag effects may contribute to some of the variation in relationships in Fig. 2.

AUTHOR CONTRIBUTIONS: R.D..S.-S. conceived the study and drafted the manuscript, G.J.E. and R.D..S.-S. led data collection with assistance from many others, C.M. and R.D..S.-S. developed the SGI with input from colleagues, C.M. and A.E.B. analysed the data, and all authors contributed to the writing.

DATA AVAILABILITY: Raw data from the Reef Life Survey program are accessible through a live data portal via the Reef Life Survey website www.reeflifesurvey.com. SGI values will be accessible through the RLS Reef Species of the World online species database by $1^{\text {st }}$ November 2020 (https://reeflifesurvey.com/species/search.php).

COMPETING INTERESTS STATEMENT: The authors declare no competing interests

ACKNOWLEDGMENTS: We thank the many Reef Life Survey (RLS) divers and scientific collaborators who assisted with field surveys, Antonia Cooper, Just Berkhout and Ella Clausius at the University of Tasmania for logistics and data management, and Dani Ceccarelli, Elizabeth Oh, Anna Cresswell, and Joe Duggan for analysis of photoquadrats. We also thank Neville Barrett, Jemina Stuart-Smith, Sue Baker and Tom Bird for further support in the development of RLS, fieldwork and concepts explored in the paper. Development of RLS was supported by the former Commonwealth Environment Research Facilities Program, while analyses were supported by the Marine Biodiversity Hub, a collaborative partnership supported through funding from the Australian Government's National Environmental Science Program, and by the Australian Research Council. Funding and support for GBR field surveys was provided by The lan 
Potter Foundation and Ningaloo surveys by the Western Australian State NRM. RLS data management is supported by Australia's integrated marine observing system (IMOS). IMOS is enabled by the national collaborative research infrastructure strategy (NCRIS). It is operated by a consortium of institutions as an unincorporated joint venture, with the University of Tasmania as lead agent.

\section{REFERENCES}

101 McKinney, M. L. \& Lockwood, J. L. Biotic homogenization: A few winners replacing many losers in the next mass extinction. Trends in Ecology and Evolution 14, 450-453 (1999).

2 Magurran, A. E., Dornelas, M., Moyes, F., Gotelli, N. J. \& McGill, B. Rapid biotic homogenization of marine fish assemblages. Nat Commun 6, 8405, doi:10.1038/ncomms9405

https://www.nature.com/articles/ncomms9405\#supplementary-information (2015).

15

20
Devictor, V. et al. Functional biotic homogenization of bird communities in disturbed landscapes. Global Ecol. Biogeogr. 17, 252-261, doi:10.1111/j.1466-8238.2007.00364.x (2008).

4 Devictor, V., Julliard, R. \& Jiguet, F. Distribution of specialist and generalist species along spatial gradients of habitat disturbance and fragmentation. Oikos 117, 507-514, doi:10.1111/j.00301299.2008.16215.x (2008).

5 Richardson, L. E., Graham, N. A. J., Pratchett, M. S., Eurich, J. G. \& Hoey, A. S. Mass coral bleaching causes biotic homogenization of reef fish assemblages. Glob Chang Biol 24, 31173129, doi:10.1111/gcb.14119 (2018).

6 Wilson, S. K. et al. Habitat utilization by coral reef fish: Implications for specialists vs. generalists in a changing environment. J. Anim. Ecol. 77, 220-228 (2008).

7 Munday, P. L. Habitat loss, resource specialization, and extinction on coral reefs. Global Change Biology 10, 1642-1647, doi:10.1111/j.1365-2486.2004.00839.x (2004).

8 Jones, G. P., McCormick, M. I., Srinivasan, M. \& Eagle, J. V. Coral decline threatens fish biodiversity in marine reserves. Proceedings of the National Academy of Sciences of the United States of America 101, 8251-8253 (2004).

9 Paddack, M. J. et al. Recent Region-wide Declines in Caribbean Reef Fish Abundance. Current Biology 19, 590-595, doi:https://doi.org/10.1016/j.cub.2009.02.041 (2009).

10 Hughes, T. P. et al. Spatial and temporal patterns of mass bleaching of corals in the Anthropocene. Science 359, 80-83, doi:10.1126/science.aan8048 (2018).

11 Hughes, T. P. et al. Coral reefs in the Anthropocene. Nature 546, 82-90,

12 Cheal, A. J., MacNeil, M. A., Emslie, M. J. \& Sweatman, H. The threat to coral reefs from more intense cyclones under climate change. Global Change Biology 23, 1511-1524, doi:10.1111/gcb.13593 (2017).

3 Oliver, E. C. J. et al. Longer and more frequent marine heatwaves over the past century. Nat Commun 9, 1324, doi:10.1038/s41467-018-03732-9 (2018).

4 Ling, S. D., Johnson, C. R., Frusher, S. D. \& Ridgway, K. R. Overfishing reduces resilience of kelp beds to climate-driven catastrophic phase shift. Proceedings of the National Academy of Sciences 106, 22341-22345, doi:10.1073/pnas.0907529106 (2009).

Sunday, J. M. et al. Species traits and climate velocity explain geographic range shifts in an ocean-warming hotspot. Ecol. Lett. 18, 944-953, doi:10.1111/ele.12474 (2015). 
16 Mair, L. et al. Abundance changes and habitat availability drive species' responses to climate change. Nature Climate Change 4, 127-131, doi:10.1038/nclimate2086 (2014).

17 Monaco, C. J. et al. Dietary generalism accelerates arrival and persistence of coral-reef fishes in their novel ranges under climate change. Global Change Biology n/a, doi:10.1111/gcb.15221. (2015).

19 Munday, P. L., Jones, G. P., Pratchett, M. S. \& Williams, A. J. Climate change and the future for coral reef fishes. Fish Fish. 9, 261-285 (2008).

Edgar, G. J. \& Stuart-Smith, R. D. Systematic global assessment of reef fish communities by the Reef Life Survey program. Scientific Data 1, 140007, doi:10.1038/sdata.2014.7 (2014).

21 Pratchett, M. S. et al. Effects of climate-induced coral bleaching on coral-reef fishes - Ecological and economic consequences. Oceanography and Marine Biology: Annual Review 46, 251-296 (2008).

Stuart-Smith, R. D., Brown, C. J., Ceccarelli, D. M. \& Edgar, G. J. Ecosystem restructuring along the Great Barrier Reef following mass coral bleaching. Nature 560, 92-96, doi:10.1038/s41586018-0359-9 (2018).

23 Feary, D. A. The influence of resource specialization on the response of reef fish to coral disturbance. Mar. Biol. 153, 153-161 (2007). relationships in coral reef fish communities. Proceedings of the Royal Society B: Biological Sciences 281, 20131993 (2014).

25 Wernberg, T. et al. Climate-driven regime shift of a temperate marine ecosystem. Science 353, 169-172, doi:10.1126/science.aad8745 (2016). shallow-water marine species. Nat Ecol Evol 1, 1846-1852, doi:10.1038/s41559-017-0353-x (2017).

27 Stuart-Smith, R. D., Edgar, G. J., Barrett, N. S., Kininmonth, S. J. \& Bates, A. E. Thermal biases and vulnerability to warming in the world's marine fauna. Nature $528,88-92$, doi:10.1038/nature16144 (2015).

28 Vergés, A. et al. Long-term empirical evidence of ocean warming leading to tropicalization of fish communities, increased herbivory, and loss of kelp. Proceedings of the National Academy of Sciences 113, 13791-13796, doi:10.1073/pnas.1610725113 (2016).

29 Booth, D. J., Figueira, W. F., Gregson, M. A., Brown, L. \& Beretta, G. Occurrence of tropical fishes in temperate southeastern Australia: Role of the East Australian Current. Estuar. Coast. Shelf Sci. 72, 102-114 (2007).

30 Feary, D. A. et al. Latitudinal shifts in coral reef fishes: why some species do and others do not shift. Fish Fish. 15, 593-615, doi:10.1111/faf.12036 (2014).

31 Guisan, A. et al. Scaling the linkage between environmental niches and functional traits for improved spatial predictions of biological communities. Global Ecol. Biogeogr. 0, doi:10.1111/geb.12967.

32 Pratchett, M. S., Hoey, A. S., Wilson, S. K., Messmer, V. \& Graham, N. A. J. Changes in Biodiversity and Functioning of Reef Fish Assemblages following Coral Bleaching and Coral Loss. Diversity 3, 424 (2011).

Johnson, C. R. et al. Climate change cascades: Shifts in oceanography, species' ranges and subtidal marine community dynamics in eastern Tasmania. J. Exp. Mar. Biol. Ecol. 400, 17-32, doi:https://doi.org/10.1016/i.jembe.2011.02.032 (2011). 
34 Dornelas, M. et al. Assemblage time series reveal biodiversity change but not systematic loss. Science 344, 296-299, doi:10.1126/science.1248484 (2014).

35 Blowes, S. A. et al. The geography of biodiversity change in marine and terrestrial assemblages. Science 366, 339-345, doi:10.1126/science.aaw1620 (2019).

Gilchrist, G. W. Specialists and Generalists in Changing Environments. I. Fitness Landscapes of Thermal Sensitivity. The American Naturalist 146, 252-270, doi:10.1086/285797 (1995).

37 Pellissier, L. et al. Quaternary coral reef refugia preserved fish diversity. Science 344, 1016-1019, doi:10.1126/science.1249853 (2014).

38 Graham, M. H., Kinlan, B. P. \& Grosberg, R. K. Post-glacial redistribution and shifts in productivity of giant kelp forests. Proceedings of the Royal Society B: Biological Sciences 277, 399-406, doi:doi:10.1098/rspb.2009.1664 (2010).

39 Hughes, T. P. et al. Global warming and recurrent mass bleaching of corals. Nature 543, 373-377, doi:10.1038/nature21707 (2017).

40 Wismer, S., Tebbett, S. B., Streit, R. P. \& Bellwood, D. R. Spatial mismatch in fish and coral loss following 2016 mass coral bleaching. Sci. Total Environ. 650, 1487-1498, doi:https://doi.org/10.1016/i.scitotenv.2018.09.114 (2019).

41 Waldock, C., Stuart-Smith, R. D., Edgar, G. J., Bird, T. J. \& Bates, A. E. The shape of abundance distributions across temperature gradients in reef fishes. Ecol. Lett. 22, 685-696, doi:10.1111/ele.13222 (2019). Mouillot, D. et al. Rare species support vulnerable functions in high-diversity ecosystems. PLoS Biol. 11, e1001569, doi:10.1371/journal.pbio.1001569 (2013).

43 Robinson, J. P. W. et al. Productive instability of coral reef fisheries after climate-driven regime shifts. Nature Ecology \& Evolution 3, 183-190, doi:10.1038/s41559-018-0715-z (2019).

44 Cresswell, A. K. et al. Translating local benthic community structure to national biogenic reef habitat types. Global Ecol. Biogeogr. 26, 1112-1125, doi:10.1111/geb.12620 (2017).

45 Edgar, G. J., Barrett, N. S. \& Stuart-Smith, R. D. Exploited reefs protected from fishing transform over decades into convservation features otherwise absent from seascapes. Ecol. Appl. 19, 1967-1974, doi:10.1890/09-0610.1 (2009).

46 Althaus, F. et al. A Standardised Vocabulary for Identifying Benthic Biota and Substrata from Underwater Imagery: The CATAMI Classification Scheme. PLOS ONE 10, e0141039, doi:10.1371/journal.pone.0141039 (2015).

47 Carmona, C. P., de Bello, F., Mason, N. W. H. \& Lepš, J. Traits Without Borders: Integrating Functional Diversity Across Scales. Trends Ecol. Evol. 31, 382-394, doi:https://doi.org/10.1016/i.tree.2016.02.003 (2016). Stuart-Smith, R. D. et al. Integrating abundance and functional traits reveals new global hotspots of fish diversity. Nature 501, 539-542, doi:10.1038/nature12529 (2013).

49 Spalding, M. D. et al. Marine ecoregions of the world: a bioregionalization of coastal and shelf areas. Bioscience 57, 573-583 (2007).

50 mapdata: Extra Map Databases. R package version 2.3.0. (2018). habitat preference of coral reef fishes: Will generalists become more specialised in a warming ocean? Global Change Biology 24, 3158-3169, doi:10.1111/gcb.14166 (2018). 


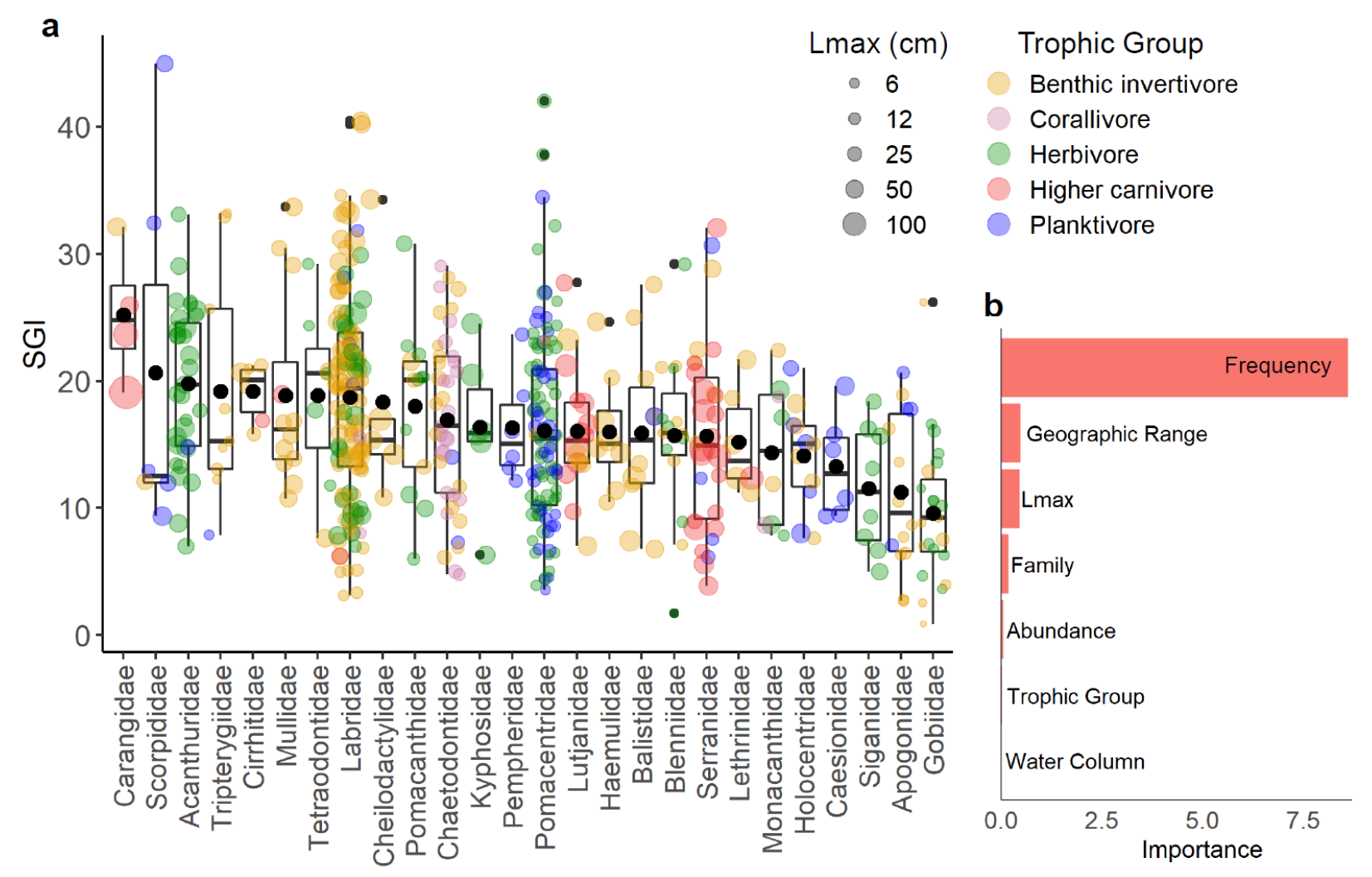

Fig. 1. Species generalisation index values (SGIs) vary among species within fish families observed on the world's reefs (a). Coloured points represent temperate and tropical reef fish species recorded on more than 70 underwater surveys across their distributions $(n=565)$, with points coloured by trophic group and symbol size scaled by the species' maximum length (Lmax). Families are ordered by mean SGI values (large black circles), while boxplots show median and inter-quartile range, $10^{\text {th }}$ and $90^{\text {th }}$ percentile whiskers and outliers. Species' frequency of occurrence was the most important predictor of their SGI amongst traits examined (b; random forest analysis; $42 \%$ variance in SGI explained). 

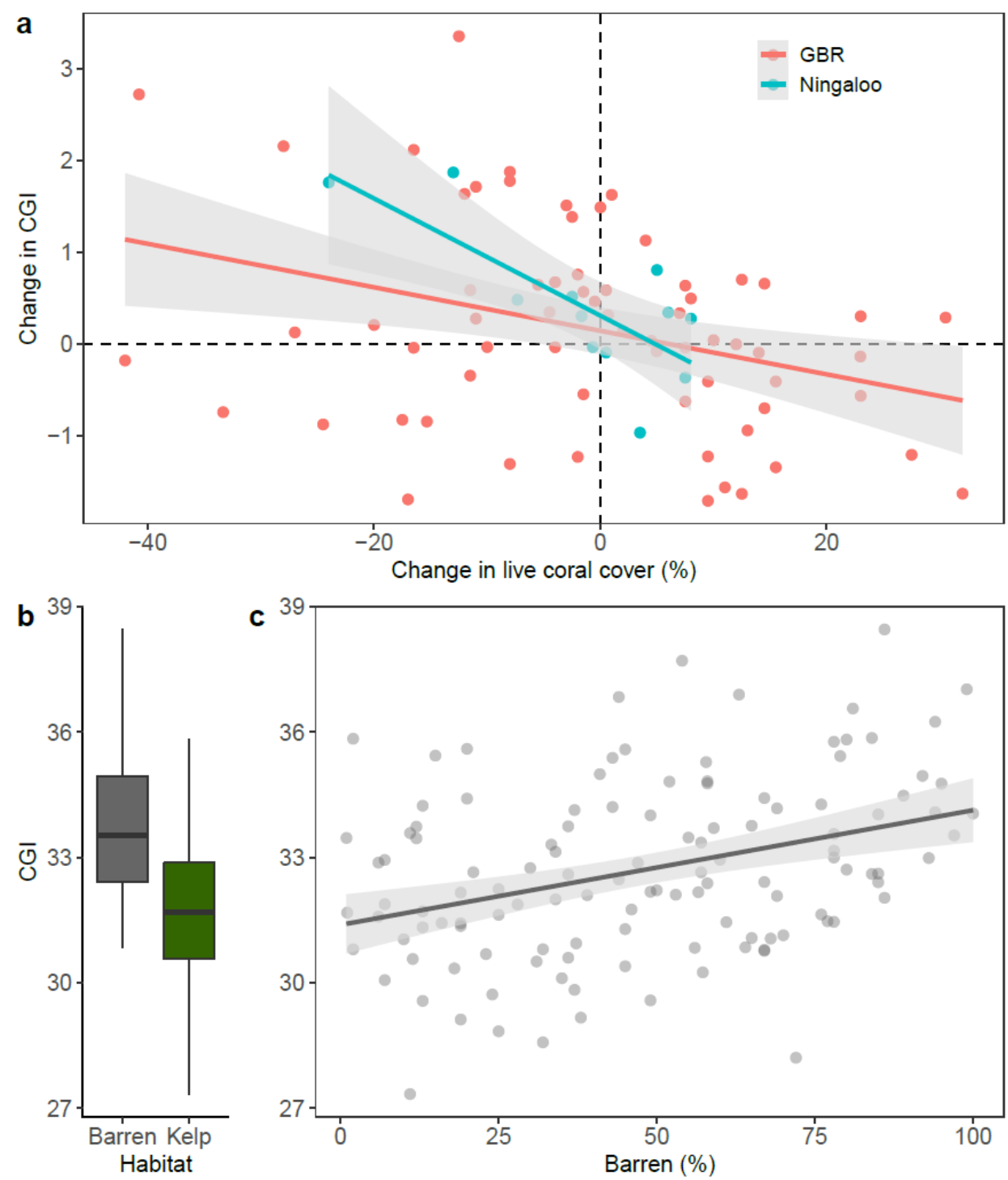

Fig. 2. Community Generalisation Index (CGI) values for reef fishes relate to loss of coral habitat and differences between kelp and urchin barrens habitat. CGI increased with coral loss at 67 sites along the Great Barrier Reef (GBR) surveyed before and after the 2016 mass bleaching event, and 12 sites surveyed at Ningaloo Reef before and after cyclone damage (a). Interspersed sites with kelp habitat support fish communities with lower CGI than sites characterised as sea urchin 'barren' habitat in southern New South Wales (b; boxplot shows median and inter-quartile range, with $10^{\text {th }}$ and $90^{\text {th }}$ percentile whiskers). CGI increases with the percentage of barrens habitat scored on surveys (c; where '\% Barren' represents the sum of bare rock and encrusting coralline algal cover. $n=123$ surveys). 


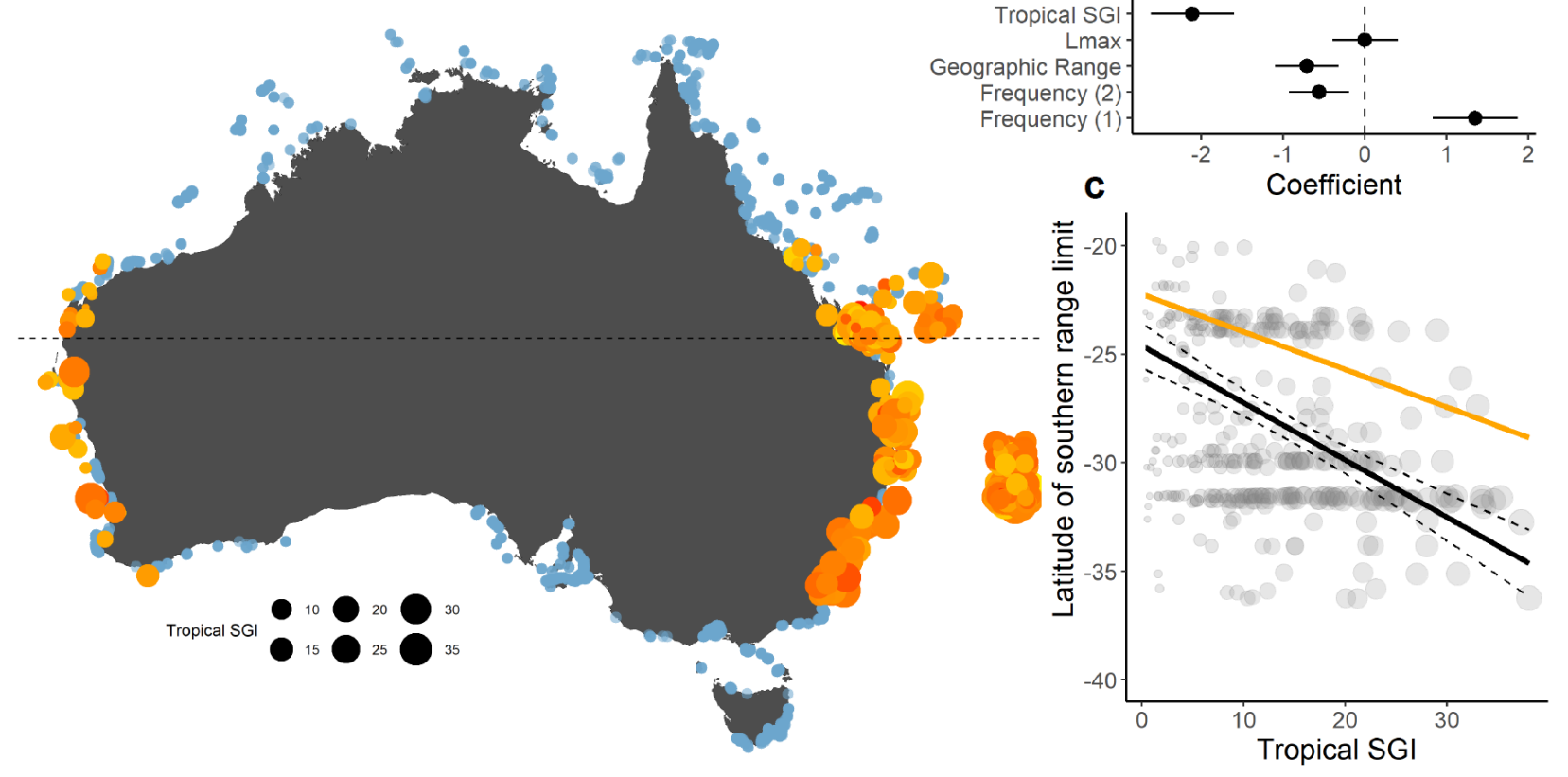

Fig. 3. Southern geographic limits of Australian tropical reef fishes (orange-yellow points in A, grey points in $\mathbf{C}, \boldsymbol{n}=\mathbf{3 6 7}$ ) relate to habitat generalisation. Point size in (a) is scaled to tropical $\mathrm{SGI}$ and colour by range size (yellow = smallest, red = largest). Blue points are all sites surveyed (i.e. where southern range limits could have been observed but were not), and the dashed horizontal line is the southern limit of the Great Barrier and Ningaloo Reefs, for reference. Species found further south have higher tropical habitat SGI values (b, c). Linear Mixed Effects model standardised estimates (and 95\% confidence intervals; b) are shown for tropical SGI and three numerical covariates (frequency of occurrence, maximum body length, and geographic range size) after accounting for the random effect of non-random representation of species within family. Frequency was fit as a polynomial term, and the coefficient for the first (1) and second (2) order terms are reported (see Methods for model details). The marginal effect of tropical SGI is plotted with species' southern latitude limits (black line; c). Dashed lines represent $95 \%$ confidence limits and the orange line represents the 80th percentile. 